\title{
Percutaneous intravenous catheter forceps biopsy in right atrial mass: two case reports and literature review
}

\author{
Lei Chang ${ }^{1}$, Chenyi Gong ${ }^{2}$, Haitao Lu³ ${ }^{3}$ Yihai Liu', Lina Kang ${ }^{2}$, Jianzhou Chen², Lian Wang ${ }^{1,2^{*}}$ (1) and Biao Xu ${ }^{1,2^{*}}$
}

\begin{abstract}
Background: Primary malignant tumors of the heart are rare. Although preoperative histological diagnosis is difficult, it has paramount value in therapeutic strategy development and prognostic estimation. Herein, we reported 2 cases of intracardiac tumors.

Cases presentation: Both patients presented to the hospital with heart-related symptoms. Echocardiography showed massive masses in the atrium and positron emission tomography-computed tomography (PET/CT) revealed hypermetabolism and invasiveness. One patient cannot take surgery due to extensive metastasis and poor condition. The other patient was primarily diagnosed with lymphoma, and surgery was not recommended. They successfully underwent intravenous atrial biopsy, and histological samples confirmed intimal sarcoma and diffuse large B cell lymphoma. Based on immunohistochemical and molecular assessments, targeted chemotherapy was administered, resulting in clinical and imaging remission at discharge.

Conclusions: Percutaneous intravenous catheter biopsy as a safe invasive test provides an accurate pathological diagnosis after imaging evaluation, and offers a therapeutic direction. Nonmalignant masses and some chemo-radiosensitive malignant tumors in the atrium could have good prognosis after targeted therapy.
\end{abstract}

Keywords: Cardiac sarcoma, Intimal sarcoma, Cardiac lymphoma, Intravenous biopsy, Case report

\section{Background}

Primary cardiac tumors (PCTs) are extremely rare, with an autopsy incidence ranging from 0.001 to $0.030 \%$ [1]. A 14-year population-based study revealed a prevalence for PCT of 1.38/100,000 [2]. A meta-analysis showed that the pooled prevalence of malignancies among patients diagnosed with PCT is 9.9\% [3]. Primary cardiac malignancies (PCMs) still have poor prognosis, and attempts to overcome diagnostic and therapeutic difficulties are needed. The majority of PCMs are sarcomas, including angiosarcoma, rhabdomyosarcoma, fibrosarcoma, and Kaposi

\footnotetext{
*Correspondence: wanglianglyy@163.com; xubiao62@nju.edu.cn ${ }^{1}$ Department of Cardiology, Nanjing Drum Tower Hospital, Clinical College of Nanjing Medical University, Nanjing 210008, Jiangsu, China Full list of author information is available at the end of the article
}

sarcoma. Mesotheliomas and primary cardiac lymphomas are the next most common primary cardiac malignancies [4]. Intimal sarcoma, a mesenchymal tumor, is the least reported primary cardiac tumor that originates from the tunica intima of large blood vessels, and rarely involves the heart [5]. PCT has multiple clinical presentations, ranging from asymptomatic detection in imaging tests to palpitation, shortness of breath, emaciation and even aborted sudden cardiac death [6]. Preoperative histological diagnosis is difficult but has paramount value in therapeutic strategy development and prognostic estimation. Herein, we successfully performed percutaneous atrial mass biopsy (PAMB), and established histological diagnosis instead of surgery. Safety, procedure and benefit for patients are key points for the extensive use of this invasive diagnostic technique. 


\section{Cases presentation}

Case 1: A 48-year-old male was presented with palpitation and chest distress after activity for 3 months. Echocardiography revealed a large mass (maximum size: $58 \mathrm{~mm} \times 40 \mathrm{~mm}$ ) attached to the lateral and posterior atrial walls, invading the inferior vena cava. Cardiac magnetic resonance (CMR) imaging showed right atrial soft tissue mass close to the tricuspid orifice with the valve leaflet (Fig. 1a). Positron emission tomographycomputed tomography (PET/CT) suggested enhanced fluorodeoxyglucose (FDG) metabolism signals in the right atrium, pericardium, mediastinum lymph nodes, and left lung nodules (Fig. 1b, c). Surgical resection was not recommended because of extensive metastasis. Totally 2 months later, the patient experienced severe chest tightness, wheezing, sitting breathing, lower limb edema, and bilateral bloody pleural effusion. Radiographic evaluations showed a right atrial mass and multiple metastatic lesions that had progressed (Fig. 2a-d).
To confirm the nature of the mass, PAMB was performed through the femoral vein, wherein three tissues were removed. The pathological analysis revealed thrombus which was consistent with imaging features. We speculated that blood flow changes caused blood turbulence. This could increase the risk of thrombus adhesion on the mass surface and influence biopsy results. After 3 weeks, the patient underwent second PAMB through the internal jugular vein. With the bedside echocardiography auxiliary positioning, three tumor tissues were removed from the atrial mass. The pathological report concluded intraepithelial metaplasia; the abnormal cell pattern showed high-grade spindle cell neoplasm with moderate atypia and focal necrosis, consistent with intimal sarcoma histologically (Fig. 3c, d). The patient was finally diagnosed with primary cardiovascular intimal sarcoma (stage IV).

Subsequently, he underwent exploratory tumor immunotherapy. The primary drug was the immune
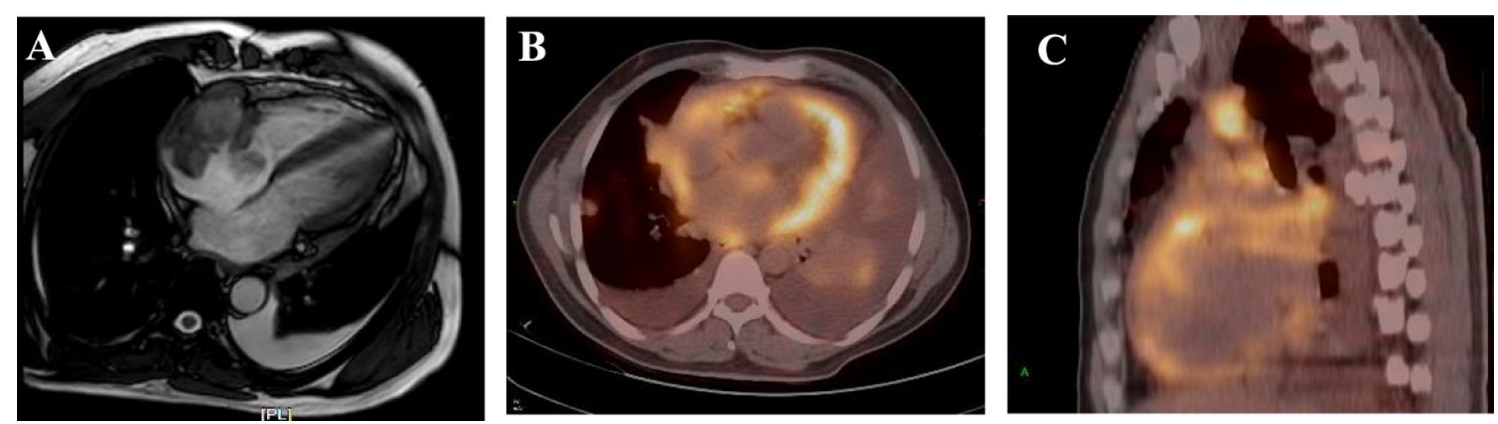

Fig. 1 CMR shows the right atrium soft tissue mass closed to the tricuspid orifice (a). PET/CT scan suggests intensive tracer uptake of the right atrial mass, pericardium, lung and mediastinal lymph nodes $(\mathbf{b}, \mathbf{c})$
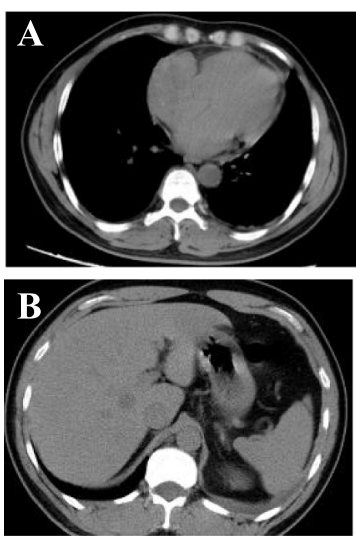

No surgical or chemotherapy

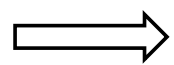

2 months
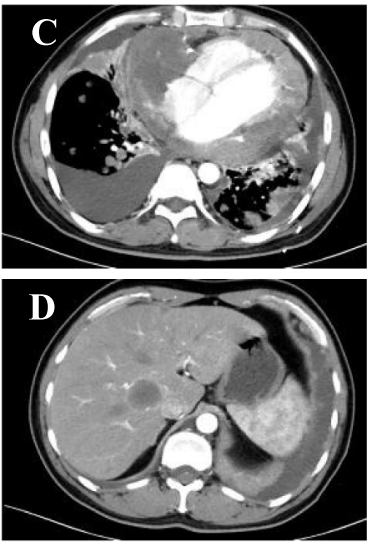

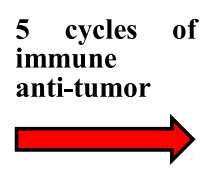

4 months
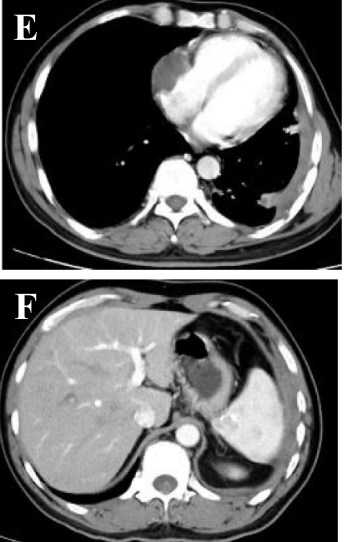

Fig. 2 CT imaging displayed right atrium mass (a) and suspected metastases in liver (b). Scan enhancement CT scan show abnormal signals in the right atrium, pericardium (c), and liver (d) in 2 months. After 5 cycles of comprehensive immune anti-tumor therapy, significant reduction is detected in the right atrial intimal sarcoma (e) and liver mass (f) dimension 

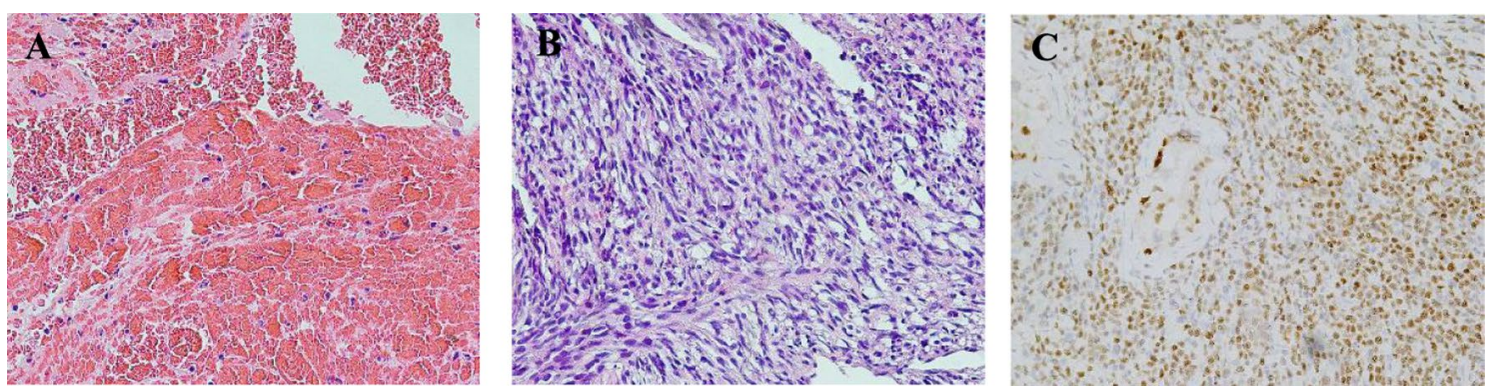

Fig. 3 The pathology of the first biopsy via femoral vein shows a large amount of cellulose and blood cells, diagnosed as thrombus (a hematoxylin and eosin, $40 \times 10)$. The pathology of the secondary biopsy through the right internal jugular vein reveals a spindle cell tumor, some areas are rich in spindle cells, with moderate atypia and focal necrosis, and the histology is consistent with intimal sarcoma (b hematoxylin and eosin, $40 \times 10$ ). Immunohistochemistry-positive neoplastic cells for PRAME (c $40 \times 10)$

checkpoint inhibitor PD-1 antibody, which could activate endogenous anti-tumor response and has confirmed efficacy in some soft-tissue sarcomas [7]. After five cycles, the patient's symptoms were relieved, and cardiac function was improved significantly. Re-examination by chest $\mathrm{CT}$ revealed a shrunk tumor in the right atrium, and the number of metastases was decreased substantially (Fig. 2e, f). At the time of drafting this manuscript, the condition of patient was stable and receiving the thirteenth cycle of immunotherapy. His health condition and life quality were improved significantly.

Case 2: A 77-year-old woman presented to our hospital with a half-month history of chest tightness and dyspnea. Sinus tachycardia and pulmonary moist rales were found on physical examination. Transthoracic echocardiography revealed an intracardiac mass (maximum size: $49 \mathrm{~mm} \times 38 \mathrm{~mm}$ ) attached to the left atrium that invaded the right atrial walls and tricuspid annulus. The atrial mass protruding into the pericardial cavity caused moderate pericardial effusion and early signs of tamponade. CMR confirmed the presence of an invasive intracardiac mass (Fig. 4a, b) and
PET-CT scan revealed high FDG uptake in the neoplasm and mediastinum lymph nodes (Fig. 4c). The patient underwent pericardium puncture drainage, and abnormal lymphocytes were found by pericardial effusion smear examination. Abnormally increased percent of heteromorphic monoclonal B lymphocytes was detected by flow cytometry. Given the right atrium invasion of the mass, and our successful experience of PAMB in the right atrium, we performed percutaneous jugular puncture and catheterization, and subsequent atrium angiography revealed a filling defect in the right atrium. Then, biopsy catheter was positioned at the neoplasm, and TTE provided multiple heart sections that confirmed the relative positions of biopsy forceps, atrial walls, and the mass. We successfully obtained 2 tissue pieces to avoid thoracotomy, without complication.

Immunohistochemical analysis of the specimen showed that the obtained tumor cells were positive for CD20, MUM1, CD5, and Bcl-2; negative for CD3, CD99, CD10 and CD30; and equivocalfor Bcl-6. $\mathrm{Ki} 67, \mathrm{Bcl}-2$, and c-MYC labeling indexes were all $90 \%$
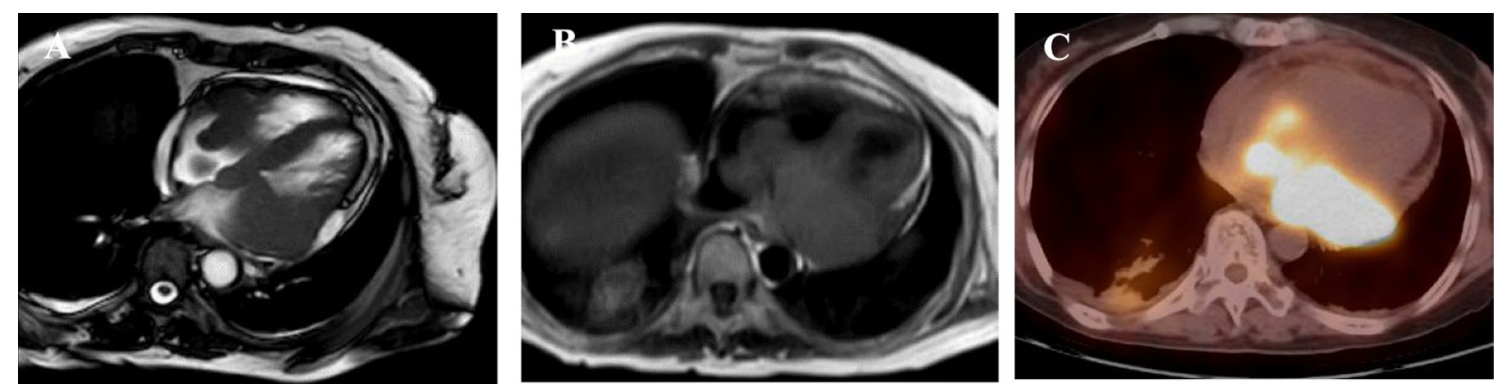

Fig. 4 CMR confirmed left atrial mass invaded right atrium $(\mathbf{a}, \mathbf{b})$ and PET-CT scan revealed high FDG uptake in atrum and mediastinal lymph nodes (c) 

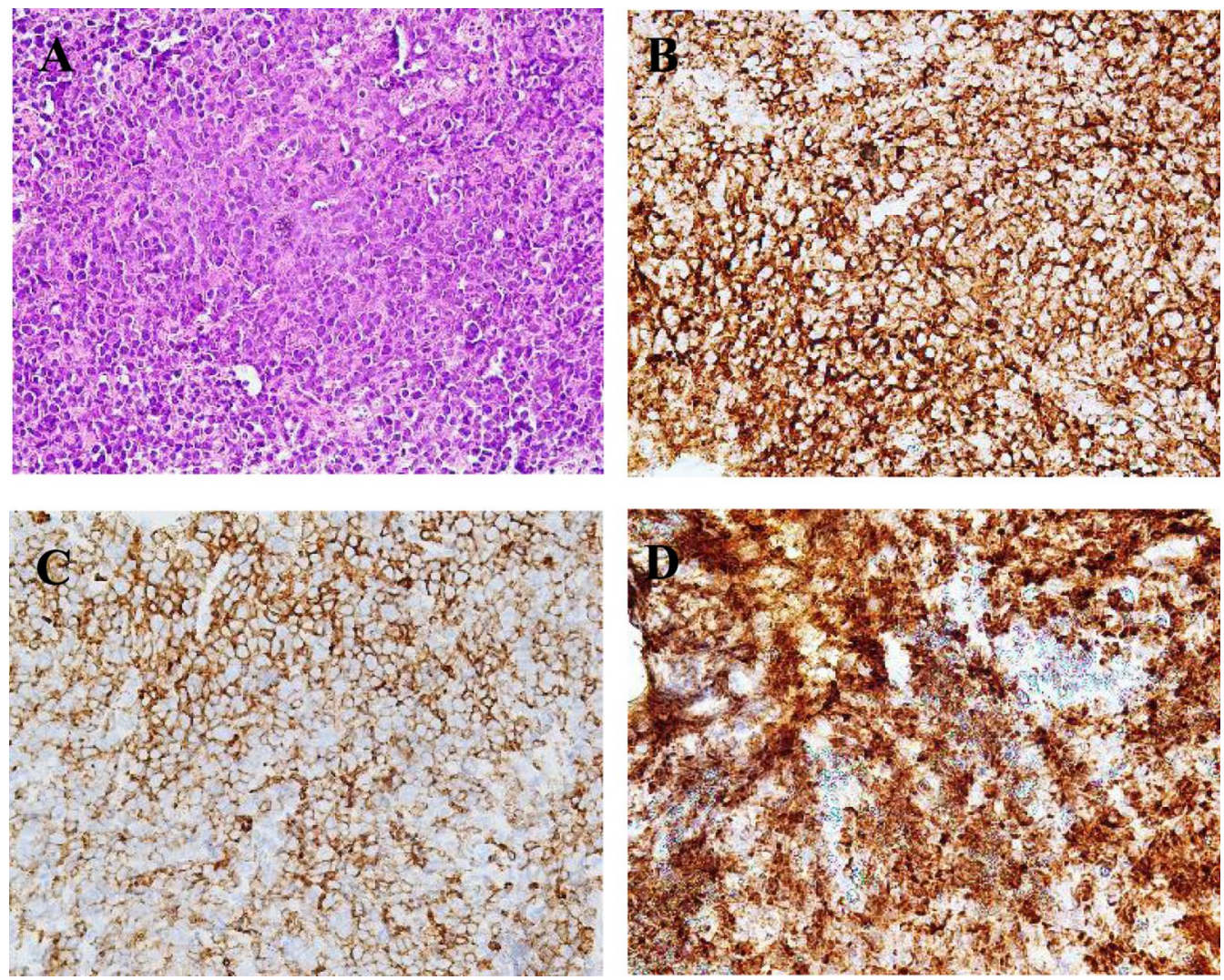

Fig. 5 The histopathological section shows diffuse growth of large lymphocytes with a small amount of apoptotic necrosis (a hematoxylin and eosin, $40 \times 10)$. Immunohistochemistry-positive neoplastic lymphoid cells for CD20 (b $40 \times 10), C D 5(\mathbf{c} \times 400)$ and Bcl2 (d $40 \times 10)$

(Fig. 5a-d). However, bone marrow biopsy showed no infiltration of abnormal lymphocytes. Then, the patient was diagnosed with cardiac diffuse large B cell lymphoma, of non-germinal center B-cell-like type. After 3 cycles of chemotherapy with R-miniCHOP (rituximab, cyclophosphamide, adriamycin, vincristine, and prednisone), the clinical symptoms were improved remarkably. Repeated echocardiography demonstrated disappearance of the intracardiac mass. PET/CT showed no area of FDG hypermetabolism in the heart or intrathoracic lymph nodes. The patient would accept the full course of treatment with continuous follow-up.

\section{Discussion and conclusions}

A recent multicenter analysis showed that PCM mainly affected adults (mean age of 53 years), and had a dismal long-term survival rate despite various modes of treatment [8]. The overall 1-year and 5-year survival rates were $45.3 \%$ and $11.5 \%$ in the latter study. Patients who underwent surgery had significantly better survival compared with those administered the non-surgical treatment. Moeri-Schimmel et al. indicated that most PCM patients who received postoperative radiotherapy had longer survival time than those who undergo only surgery [9]. Multi-institutional data from the National Cancer Database also confirmed that stage III PCM patients who undergo surgery and receive perioperative chemotherapy have better survival compared with those who did not receive this treatment [8]. Although surgical resection remains the first treatment choice for cardiac sarcoma [1], radiotherapy and chemotherapy are increasingly important. Thus, individualized radiotherapy and chemotherapy should be recommended in inoperable patients. A prospective study of right-sided tumors showed that neoadjuvant chemotherapy reduces the tumor burden, improving resectability and survival in complex cardiac tumors [10].

Although intracardiac mass is considered as a dangerous signal of PCM, it should be noticed that a neoplasm located in the atrium usually causes less 
systolic dysfunction and ventricular arrhythmia. Moreover, benign lesions such as thrombus and myxoma, accounting for a large proportion of atrial masses, but seldom appear in ventricles. Therefore, the criticality of atrial mass is different from that of ventricular mass. For atrial mass, more effort is needed to enable precise diagnosis, especially histological diagnosis. Echocardiography, CT, CMR, and PET/CT imaging could help in the preliminary differentiation between malignant and benign tumors based on tumor shape, aggressiveness, and radioactive material intake $[11,12]$. However, several intra-atrial PCMs firstly misdiagnosed as benign tumors or incompletely resected may relapse in a short time [13]. Generally, histological diagnosis before tumor therapy could avoid this situation and help differentiate primary sarcomas from other malignancies (such as lymphomas that do not require surgery). After that, the patients could be administered the best-personalized multimodality treatment [14]. In patients with potential resectable cardiac sarcomas but limited by poor cardiac and pulmonary functions [15], pathological results could facilitate neoadjuvant chemotherapy to improve the basic condition of patients and create opportunities or strengthen the effect of surgical resection.

The endomyocardial biopsy technology has been widely used in cardiomyopathy, and has been extended to ventricle mass biopsy. By contrast, atrial biopsy has not been promoted for some reason. Atrial biopsy means thinner cardiac muscle walls, closer intervention to the relatively fragile vein, and higher risk of thrombus detachment. In 1989, Gosalakkal and Sugrue [16] firstly reported atrium biopsy and removed the tumor tissue from the cardiac chamber. To further evaluate the safety and efficacy of percutaneous atrial mass biopsy, we analyzed 51 cases in 47 articles and our 2 PAMB cases (Table 1). Most patients had undergone simple right atrial lump biopsy, while 3 cases underwent transseptal left atrial biopsy. Of all cases, only 1 patient reported potential complications post-biopsy [17]. The latter patient developed severe hypotensive shock after the procedure. After fluid resuscitation and treatment with vasoactive agents, the shock symptoms were stabilized. Since no obvious signs of perforation and rupture of the atrium or inferior vena cava were noted, mechanical damage to the heart caused by biopsy was not considered.

Among 37 cases with reported malignant tumors, lymphomas accounted for the largest proportion (15/37), followed by soft tissue sarcomas (13/37), and 9/37 reported metastatic tumors. Table 2 shows their prognoses differed from mass location and pathological results. In all patients, atrial lymphoma patients had the best prognosis, including 11 patients who presented clinical remission or even cured after receiving corresponding chemotherapy; the prognoses of another 4 lymphoma patients were not reported. Most atrial soft-tissue sarcoma patients cannot tolerate surgery and radiotherapy due to heart-related symptoms; 6 patients achieved clinical remission and 3 had tumor exacerbation in short-term visits. Of another 4 patients diagnosed with atrial thrombosis, 2 had satisfactory prognosis, and 1 diagnosed with angiosarcoma by subsequent surgical specimens died of tumor recurrence and metastasis [34]. In addition, a patient pathologically diagnosed with an IgG4-related disease received hormone therapy and showed good prognosis [61].

For various spatial atrial mass locations, different vessel approaches and ultrasonic guidance methods could be selected in PAMB. Biopsy has been performed via the internal jugular, femoral and subclavian veins. Transesophageal echocardiography (TEE) is highly effective in mass locating and bioptome guidance. Hence, about $50 \%$ of physicians use TEE as a supplementary tool to digital subtraction angiography (DSA). Transthoracic echocardiography (TTE) is more convenient but provides less location information compared with TEE, thereby necessitating highly experienced doctors. Intracardiac echocardiography (ICE) can provide the most precise intra-atrial imaging and structures, but requires an extra-intravenous ultrasound catheter, which limits its application; only about $33 \%$ of physicians utilize this method. Table 3 shows the proportion of different guiding methods in all transvenous atrium biopsy cases.

Percutaneous intravenous catheter biopsy provided accurate pathological diagnosis after imaging evaluation, avoiding unnecessary surgical excision and reducing medical resource waste. Benign tumors, thrombus, bacterial vegetation, and lymphoma detected by PAMB could be cured. Nevertheless, when pathological biopsy results suggest thrombus but do not correspond to clinical symptoms, doctors should consider the possibility of false negatives. Multiple biopsies from different vessel approaches and varied mass locations might reduce the probability of misleading results. However, whether biopsies are beneficial to PCM patients is currently controversial. Ryo et al. [34] speculated that intravenous cardiac biopsy and subsequent chemotherapy is not beneficial to cardiac sarcoma patients. The core challenge is the effectiveness of subsequent anti-tumor therapy. Significantly, with the development of antitumor therapy, appropriate treatments have proved effective. PAMB as a safe, effective, and minimally invasive procedure for histological diagnosis before surgical resection would be applied increased comprehensively. 
Table1 Atrial mass cases diagnosed by intravenous biopsy

\begin{tabular}{|c|c|c|c|c|c|c|c|c|c|}
\hline Age & Sex & $\begin{array}{l}\text { Biopsy } \\
\text { location }\end{array}$ & $\begin{array}{l}\text { Transvenous } \\
\text { pathway }\end{array}$ & $\begin{array}{l}\text { Guidance } \\
\text { Method }\end{array}$ & $\begin{array}{l}\text { Presenting } \\
\text { reasons }\end{array}$ & $\begin{array}{l}\text { Pathological } \\
\text { diagnosis }\end{array}$ & Treatment & Outcome & PMID \\
\hline 14 & Male & RA & Femoral vein & DSA + TEE & $\begin{array}{l}\text { Chest pain, } \\
\text { cough, and } \\
\text { hemoptysis }\end{array}$ & / & / & I & $2,334,836[18]$ \\
\hline 46 & Female & RA & Jugular vein & DSA & $\begin{array}{l}\text { Symptoms of } \\
\text { Upper respira- } \\
\text { tory infection }\end{array}$ & $\begin{array}{l}\text { Metastases of } \\
\text { melanoma }\end{array}$ & / & / & $2,765,329[16]$ \\
\hline 83 & Female & RA & Femoral vein & DSA & $\begin{array}{l}\text { Dyspnea and } \\
\text { hypodynamia }\end{array}$ & $\begin{array}{l}\text { Papillary fibroe- } \\
\text { lastoma }\end{array}$ & $\begin{array}{l}\text { Symptomatic } \\
\text { treatment }\end{array}$ & / & 2,816,690 [19] \\
\hline 69 & Female & RA & Femoral vein & DSA + TEE & $\begin{array}{l}\text { Dyspnea, } \\
\text { edema, and } \\
\text { syncope }\end{array}$ & Thrombus & $\begin{array}{l}\text { Surgical resec- } \\
\text { tion }\end{array}$ & Remission & $7,499,910$ [20] \\
\hline 52 & Female & RA & Femoral vein & DSA + TEE & $\begin{array}{l}\text { History of cir- } \\
\text { rhosis }\end{array}$ & $\begin{array}{l}\text { Metastases of } \\
\text { liver cancer }\end{array}$ & / & Deceased & $8,131,572[21]$ \\
\hline 69 & Male & RA & Femoral vein & $\mathrm{DSA}+\mathrm{TEE}$ & $\begin{array}{l}\text { Dyspnea, } \\
\text { cough, and } \\
\text { hemoptysis }\end{array}$ & $\begin{array}{l}\text { Metastases of } \\
\text { melanoma }\end{array}$ & / & I & $8,154,436[22]$ \\
\hline 69 & Female & RA & Jugular vein & $\mathrm{DSA}+\mathrm{TEE}$ & $\begin{array}{l}\text { Dyspnea, } \\
\text { dehydration, } \\
\text { and ventricular } \\
\text { tachycardia }\end{array}$ & Thrombus & $\begin{array}{l}\text { Symptomatic } \\
\text { treatment }\end{array}$ & / & $8,365,328$ [23] \\
\hline 62 & Male & RA & Jugular vein & $\mathrm{DSA}+\mathrm{TEE}$ & $\begin{array}{l}\text { Edema and } \\
\text { hepatic } \\
\text { encephalopa- } \\
\text { thy }\end{array}$ & $\begin{array}{l}\text { Metastatic } \\
\text { adenocarci- } \\
\text { noma }\end{array}$ & / & Deceased & $8,498,328$ [24] \\
\hline 73 & Female & RA & Jugular vein & $\mathrm{DSA}+\mathrm{TEE}$ & $\begin{array}{l}\text { Edema, cough, } \\
\text { fever, and } \\
\text { dyspnea }\end{array}$ & Lymphoma & Chemotherapy & Remission & $8,579,042[25]$ \\
\hline 69 & Male & RA & Femoral vein & $D S A+I C E$ & Flu symptoms & $\begin{array}{l}\text { Metastasis of } \\
\text { lung cancer }\end{array}$ & / & / & $8,611,294$ [26] \\
\hline 73 & Male & RA & Jugular vein & DSA + TEE & Asymptomatic & $\begin{array}{l}\text { Metastases of } \\
\text { melanoma }\end{array}$ & Chemotherapy & / & $8,945,488$ [27] \\
\hline 35 & Male & RA & Femoral vein & $\mathrm{DSA}+\mathrm{TEE}$ & $\begin{array}{l}\text { Dyspnea and } \\
\text { cough }\end{array}$ & Angiosarcoma & Chemotherapy & Remission & $8,974,823[28]$ \\
\hline / & I & RA & Jugular vein & $\mathrm{DSA}+\mathrm{TEE}$ & / & Angiosarcoma & / & l & $9,070,559[29]$ \\
\hline 62 & Male & RA & Jugular vein & $\mathrm{DSA}+\mathrm{TEE}$ & $\begin{array}{l}\text { Dyspnea, chest } \\
\text { pain and }\end{array}$ & $\begin{array}{l}\text { Undifferenti- } \\
\text { ated sarcoma }\end{array}$ & Chemotherapy & Deceased & $9,339,428[30]$ \\
\hline 66 & I & $\mathrm{RA}+\mathrm{AS}$ & Femoral vein & DSA + TEE & $\begin{array}{l}\text { Myocardial } \\
\text { infarction }\end{array}$ & Lymphoma & Chemotherapy & / & $9,454,452[31]$ \\
\hline 64 & Female & RA & l & $\mathrm{DSA}+\mathrm{TEE}$ & Dizziness & Lymphoma & / & / & $9,487,479[32]$ \\
\hline 47 & Male & RA & Jugular vein & DSA+TTE & $\begin{array}{l}\text { Dyspnea, } \\
\text { edema and } \\
\text { pleural effusion }\end{array}$ & Lymphoma & Chemotherapy & / & $9,829,904$ [33] \\
\hline 50 & Male & RA & / & DSA + TEE & $\begin{array}{l}\text { Orthopnea, } \\
\text { fever, and pleu- } \\
\text { ral effusion }\end{array}$ & Thrombus & $\begin{array}{l}\text { Surgical resec- } \\
\text { tion }\end{array}$ & Deceased & $9,932,633[34]$ \\
\hline 39 & Female & / & / & / & / & Myxoma & / & / & $10,231,677[35]$ \\
\hline / & / & RA & / & / & / & / & / & / & $10,763,354[36]$ \\
\hline 77 & Female & RA & / & $\mathrm{DSA}+\mathrm{TEE}$ & $\begin{array}{l}\text { Dyspnea, } \\
\text { edema and } \\
\text { hypodynamia }\end{array}$ & Lymphoma & $\begin{array}{l}\text { Chemoradio- } \\
\text { therapy }\end{array}$ & Remission & $10,790,358[37]$ \\
\hline 75 & Male & RA & Femoral vein & $\mathrm{DSA}+\mathrm{TEE}$ & Hemoptysis & Lymphoma & Chemotherapy & Remission & $10,842,397[38]$ \\
\hline 79 & Female & RA & Femoral vein & $D S A+I C E$ & $\begin{array}{l}\text { Dyspnea and } \\
\text { edema }\end{array}$ & Neuroblastoma & / & / & $10,952,166[39]$ \\
\hline I & / & LA & / & $\mathrm{DSA}+\mathrm{TEE}$ & / & Atrial sarcoma & / & / & $11,223,492[40]$ \\
\hline 52 & Male & RA & Jugular vein & $\mathrm{DSA}+\mathrm{TEE}$ & Epigastric ache & Angiosarcoma & Chemotherapy & Remission & $12,019,433[41]$ \\
\hline 62 & Male & RA & Femoral vein & $\mathrm{DSA}+\mathrm{TEE}$ & $\begin{array}{l}\text { Dyspnea and } \\
\text { dizziness }\end{array}$ & Lymphoma & Chemotherapy & Remission & $12,848,708[42]$ \\
\hline
\end{tabular}


Table1 (continued)

\begin{tabular}{|c|c|c|c|c|c|c|c|c|c|}
\hline Age & Sex & $\begin{array}{l}\text { Biopsy } \\
\text { location }\end{array}$ & $\begin{array}{l}\text { Transvenous } \\
\text { pathway }\end{array}$ & $\begin{array}{l}\text { Guidance } \\
\text { Method }\end{array}$ & $\begin{array}{l}\text { Presenting } \\
\text { reasons }\end{array}$ & $\begin{array}{l}\text { Pathological } \\
\text { diagnosis }\end{array}$ & Treatment & Outcome & PMID \\
\hline 64 & Male & $\mathrm{RA}$ & Jugular vein & $\mathrm{DSA}+\mathrm{TEE}$ & Heart failure & Lymphoma & Chemotherapy & Remission & $14,622,547[43]$ \\
\hline 52 & Male & RA & Jugular vein & DSA + TEE & $\begin{array}{l}\text { Dyspnea, chest } \\
\text { pain, and } \\
\text { edema }\end{array}$ & $\begin{array}{l}\text { Metastases of } \\
\text { liver cancer }\end{array}$ & $\begin{array}{l}\text { Symptomatic } \\
\text { treatment }\end{array}$ & Deceased & $15,546,373$ [44] \\
\hline 63 & Female & $\mathrm{RA}$ & Jugular vein & $D S A+I C E$ & $\begin{array}{l}\text { Dyspnea } \\
\text { and centrum } \\
\text { fracture }\end{array}$ & Lipomyoma & $\begin{array}{l}\text { Expectant treat- } \\
\text { ment }\end{array}$ & / & $16,880,106[45]$ \\
\hline 61 & Male & $\mathrm{RA}$ & $\begin{array}{l}\text { Subclavian } \\
\text { vein for biopsy, } \\
\text { femoral vein } \\
\text { for ICE }\end{array}$ & $D S A+I C E$ & $\begin{array}{l}\text { Ventricular } \\
\text { tachycardia }\end{array}$ & Lymphoma & / & / & $17,015,040[46]$ \\
\hline 38 & Male & $\mathrm{RA}$ & / & DSA + TTE & Dyspnea & Lymphoma & Chemotherapy & Remission & $17,383,751[47]$ \\
\hline 52 & Male & RA & Femoral vein & $D S A+I C E$ & $\begin{array}{l}\text { Cardiac tam- } \\
\text { ponade }\end{array}$ & $\begin{array}{l}\text { Granulocytic } \\
\text { sarcoma }\end{array}$ & $\begin{array}{l}\text { Chemora- } \\
\text { diotherapy and } \\
\text { hematopoietic } \\
\text { stem cell trans- } \\
\text { plantation }\end{array}$ & Remission & $18,498,027$ [48] \\
\hline 56 & Female & / & / & / & $\begin{array}{l}\text { Repeated peri- } \\
\text { cardial effusion }\end{array}$ & / & / & / & $18,805,775[49]$ \\
\hline 67 & Female & $\mathrm{RA}$ & $\begin{array}{l}\text { Jugular vein for } \\
\text { biopsy, femoral } \\
\text { vein for ICE }\end{array}$ & $D S A+I C E$ & $\begin{array}{l}\text { The discomfort } \\
\text { of the precor- } \\
\text { dial area }\end{array}$ & Paragangliomas & $\begin{array}{l}\text { Surgical resec- } \\
\text { tion }\end{array}$ & / & $18,818,096$ [17] \\
\hline 47 & Female & $\mathrm{RA}$ & $\begin{array}{l}\text { Jugular vein for } \\
\text { biopsy, femoral } \\
\text { vein for ICE }\end{array}$ & $D S A+I C E$ & $\begin{array}{l}\text { Hypodynamia } \\
\text { and atrial fibril- } \\
\text { lation }\end{array}$ & Paragangliomas & $\begin{array}{l}\text { Surgical resec- } \\
\text { tion }\end{array}$ & / & 18,818,096 [17] \\
\hline 30 & Male & RA/IVC & $\begin{array}{l}\text { Jugular vein for } \\
\text { biopsy, femoral } \\
\text { vein for ICE }\end{array}$ & $D S A+I C E$ & $\begin{array}{l}\text { Polyserositis } \\
\text { and edema } \\
\text { after heart } \\
\text { transplant }\end{array}$ & $\begin{array}{l}\text { Bacterial } \\
\text { emboli }\end{array}$ & $\begin{array}{l}\text { Surgical resec- } \\
\text { tion }\end{array}$ & Remission & $18,818,096$ [17] \\
\hline 70 & Male & RA/IVC & / & / & $\begin{array}{l}\text { Ascites after } \\
\text { heart transplan- } \\
\text { tation }\end{array}$ & $\begin{array}{l}\text { Cardiac amyloi- } \\
\text { dosis }\end{array}$ & $\begin{array}{l}\text { Symptomatic } \\
\text { treatment }\end{array}$ & Deceased & 18,818,096 [17] \\
\hline 63 & Female & RA & $\begin{array}{l}\text { Jugular vein for } \\
\text { biopsy, femoral } \\
\text { vein for ICE }\end{array}$ & $D S A+I C E$ & $\begin{array}{l}\text { Chest discom- } \\
\text { fort and edema }\end{array}$ & Lymphoma & Chemotherapy & Remission & $19,057,087[50]$ \\
\hline 59 & Male & $\mathrm{RA}$ & / & DSA + TEE & $\begin{array}{l}\text { Dyspnea, } \\
\text { cough, chest } \\
\text { discomfort, } \\
\text { fever and hypo- } \\
\text { dynamia }\end{array}$ & Lymphoma & Chemotherapy & Remission & $19,142,595[51]$ \\
\hline 64 & Female & RA & / & DSA + TTE & $\begin{array}{l}\text { Dizziness and } \\
\text { history of } \\
\text { endometrial } \\
\text { carcinoma }\end{array}$ & $\begin{array}{l}\text { Metastases of } \\
\text { endometrial } \\
\text { adenocarci- } \\
\text { noma }\end{array}$ & / & & $20,027,104[52]$ \\
\hline 22 & Female & RA & / & $D S A+I C E$ & $\begin{array}{l}\text { Dyspnea and } \\
\text { syncope }\end{array}$ & Angiosarcoma & Chemotherapy & / & $20,585,357$ [53] \\
\hline 57 & Female & RA & / & DSA + TEE & $\begin{array}{l}\text { Dyspnea and } \\
\text { back pain }\end{array}$ & Intimal sarcoma & Chemotherapy & Remission & $20,966,612[54]$ \\
\hline 44 & Male & RA & $\begin{array}{l}\text { Jugular vein for } \\
\text { biopsy, femoral } \\
\text { vein for ICE }\end{array}$ & $D S A+I C E$ & Dyspnea & Lymphoma & Chemotherapy & Remission & $22,576,384[55]$ \\
\hline 31 & Male & LA & / & $\mathrm{DSA}+\mathrm{TEE}$ & $\begin{array}{l}\text { Dyspnea and } \\
\text { hypodynamia }\end{array}$ & $\begin{array}{l}\text { Poorly differen- } \\
\text { tiated sarcoma }\end{array}$ & Chemotherapy & Deceased & $23,109,774[56]$ \\
\hline 46 & Male & RA & Femoral vein & $D S A+I C E$ & $\begin{array}{l}\text { History of } \\
\text { myxoma }\end{array}$ & Myxoma & $\begin{array}{l}\text { Expectant treat- } \\
\text { ment }\end{array}$ & Remission & $25,240,574$ [57] \\
\hline 53 & Male & RA & $\begin{array}{l}\text { Right femoral } \\
\text { vein for biopsy, } \\
\text { left femoral vein } \\
\text { for ICE }\end{array}$ & $D S A+I C E$ & $\begin{array}{l}\text { Dyspnea and } \\
\text { weight loss }\end{array}$ & $\begin{array}{l}\text { Metastases of } \\
\text { lung cancer }\end{array}$ & Chemotherapy & Deceased & $25,810,740[58]$ \\
\hline
\end{tabular}


Table1 (continued)

\begin{tabular}{|c|c|c|c|c|c|c|c|c|c|}
\hline Age & Sex & $\begin{array}{l}\text { Biopsy } \\
\text { location }\end{array}$ & $\begin{array}{l}\text { Transvenous } \\
\text { pathway }\end{array}$ & $\begin{array}{l}\text { Guidance } \\
\text { Method }\end{array}$ & $\begin{array}{l}\text { Presenting } \\
\text { reasons }\end{array}$ & $\begin{array}{l}\text { Pathological } \\
\text { diagnosis }\end{array}$ & Treatment & Outcome & PMID \\
\hline 51 & Female & RA & / & $D S A+I C E$ & $\begin{array}{l}\text { Dyspnea and } \\
\text { edema }\end{array}$ & Angiosarcoma & Chemotherapy & Deceased & $25,810,740[58]$ \\
\hline 47 & Female & RA & Femoral vein & $D S A+I C E$ & $\begin{array}{l}\text { Neoadjuvant } \\
\text { chemotherapy } \\
\text { for breast } \\
\text { cancer }\end{array}$ & Thrombus & $\begin{array}{l}\text { Surgical resec- } \\
\text { tion }\end{array}$ & Remission & $27,068,834[59]$ \\
\hline 59 & Male & LA & / & DSA + TEE & $\begin{array}{l}\text { Fever, weight } \\
\text { loss, and history } \\
\text { of HIV infection }\end{array}$ & Lymphoma & Chemotherapy & Remission & $31,020,117[60]$ \\
\hline 61 & Male & RA & / & $D S A+I C E$ & $\begin{array}{l}\text { Jaundice, } \\
\text { hypodynamia } \\
\text { and diagnosed } \\
\text { as lgG4/related } \\
\text { disease }\end{array}$ & $\begin{array}{l}\text { lgG4/related } \\
\text { disease }\end{array}$ & $\begin{array}{l}\text { Hormono- } \\
\text { therapy }\end{array}$ & Remission & $31,118,383[61]$ \\
\hline 47 & Female & RA & $\begin{array}{l}\text { Jugular vein for } \\
\text { biopsy, femoral } \\
\text { vein for ICE }\end{array}$ & $D S A+I C E$ & $\begin{array}{l}\text { Cough and } \\
\text { pulmonary } \\
\text { nodules }\end{array}$ & Angiosarcoma & Chemotherapy & Remission & $32,874,873[62]$ \\
\hline 48 & Male & $\mathrm{RA}$ & $\begin{array}{l}\text { Femoral vein } \\
\text { and jugular vein }\end{array}$ & DSA + TTE & $\begin{array}{l}\text { Palpitation and } \\
\text { chest discom- } \\
\text { fort }\end{array}$ & Intimal sarcoma & Chemotherapy & Remission & Present case \\
\hline 77 & Female & RA & Jugular vein & DSA + TTE & $\begin{array}{l}\text { Dyspnea and } \\
\text { chest discom- } \\
\text { fort }\end{array}$ & Lymphoma & Chemotherapy & Remission & Present case \\
\hline
\end{tabular}

Table 2 Prognosis of patients differs with respect to the mass locations and the pathological results

\begin{tabular}{|c|c|c|c|c|}
\hline Neoplasm location and pathology & Overall response & Progressive disease & Not mentioned & Total \\
\hline All cases & 22 & 9 & 22 & 53 \\
\hline \multicolumn{5}{|l|}{ First attack symptom } \\
\hline Related to the mass & 17 & 8 & 13 & 38 \\
\hline Unrelated to the mass & 5 & 1 & 4 & 10 \\
\hline No discomfort or not mentioned & 0 & 0 & 5 & 5 \\
\hline \multicolumn{5}{|l|}{ Biopsy location } \\
\hline Right atrium or atrial septum & 21 & 8 & 21 & 50 \\
\hline Involved left atrium & 1 & 1 & 1 & 3 \\
\hline \multicolumn{5}{|l|}{ Malignant tumor } \\
\hline Lymphoma & 11 & 0 & 4 & 15 \\
\hline Metastatic tumor & 0 & 4 & 5 & 9 \\
\hline Soft-tissue sarcoma & 6 & 3 & 4 & 13 \\
\hline \multicolumn{5}{|l|}{ Benign tumor } \\
\hline Lipomyoma & 0 & 0 & 1 & 1 \\
\hline Myxoma & 1 & 0 & 1 & 2 \\
\hline Paraganglioma & 0 & 0 & 2 & 2 \\
\hline Fibroma & 0 & 0 & 1 & 1 \\
\hline \multicolumn{5}{|l|}{ Others } \\
\hline Thrombus & 2 & 1 & 1 & 4 \\
\hline Bacterial vegetation & 1 & 0 & 0 & 1 \\
\hline IgG4-related diseases & 1 & 0 & 0 & 1 \\
\hline Myocardial amyloidosis & 0 & 1 & 0 & 1 \\
\hline Not mentioned & 0 & 0 & 3 & 3 \\
\hline
\end{tabular}

The overall response means patients' clinical symptoms were relieved and/or healed, or the tumor volume was reduced. Progressive disease means patients could not benefit from the treatment, it was worsened, or led to death 
Table 3 Proportion of different guiding methods in all transvenous atrium biopsy cases

\begin{tabular}{llllll}
\hline & \multicolumn{3}{l}{ Reported imaging and guidance method } & \multirow{2}{*}{$\begin{array}{l}\text { Not } \\
\text { mentioned }\end{array}$} \\
\cline { 2 - 4 } & $\begin{array}{l}\text { DSA } \\
\text { only }\end{array}$ & TTE+DSA & TEE+DSA & ICE+DSA & \\
\hline $\begin{array}{l}\text { Num- } \\
\text { ber of } \\
\text { cases }\end{array}$ & 2 & 6 & 24 & 17 & 4 \\
$\begin{array}{l}\text { Propor- } \\
\text { tion }\end{array}$ & $4.1 \%$ & $12.2 \%$ & $49.0 \%$ & $34.7 \%$ & \\
\hline
\end{tabular}

\section{Abbreviations}

PCT: Primary cardiac tumor; PCM: Primary cardiac malignancy; PET/CT: Positron emission tomography-computed tomography; CMR: Cardiac magnetic resonance; FDG: Fluorodeoxyglucose; PAMB: Percutaneous atrial mass biopsy; TEE: Transesophageal echocardiography; TTE: Transthoracic echocardiography; ICE: Intracardiac echocardiography; DSA: Digital subtraction angiography.

\section{Acknowledgements}

Not applicable.

\section{Authors' contributions}

LC: data review and manuscript writing. CYG: data collection and manuscript writing. HTL: data collection and management. YHL: manuscript writing. LNK: manuscript revision and data review. JZC: data review and manuscript review. LW and BX: biopsy and study design. All authors have read and approved the final manuscript.

\section{Funding}

This study was supported by grants from the Natural Science Foundation of China (81870204), and Medical Science and technology development Foundation, Nanjing Department of Health (ZKX19017). No funding body participated in the design of the study and the collection, analysis, and interpretation of data and the writing the manuscript.

\section{Availability of data and materials}

The information and data of the 2 patients were acquired from the Hospital Information System are not publicly available due to the protection of individual privacy. The literature review datasets analyzed in the current study are available from the corresponding author on reasonable request.

\section{Declarations}

\section{Ethics approval and consent to participate}

The study was approved by the Medical Ethics Committee of Nanjing Drum Tower Hospital, Medical School of Nanjing University (2019-190-01). Written informed consent was obtained from the patients for the publication of this report and any accompanying images. A copy of the written consent is available for review at any time.

\section{Consent for publication}

All authors declared the patient has signed written informed consent for publication of clinical, radiological, and biological data.

\section{Competing interests}

The authors declare that they have no competing interests.

\section{Author details}

${ }^{1}$ Department of Cardiology, Nanjing Drum Tower Hospital, Clinical College of Nanjing Medical University, Nanjing 210008, Jiangsu, China. ${ }^{2}$ Department of Cardiology, Nanjing Drum Tower Hospital, Nanjing University Medical School, Nanjing 210008, Jiangsu, China. ${ }^{3}$ Department of Cardiology, Anhui Sixian Peoples Hospital, Suzhou 234300, Anhui, China.
Received: 12 October 2021 Accepted: 10 February 2022

Published online: 20 February 2022

\section{References}

1. Butany J, Nair V, Naseemuddin A, Nair GM, Catton C, Yau T. Cardiac tumours: diagnosis and management. Lancet Oncol. 2005;6(4):219-28.

2. Cresti A, Chiavarelli M, Glauber M, Tanganelli P, Scalese M, Cesareo F, Guerrini F, Capati E, Focardi M, Severi S. Incidence rate of primary cardiac tumors: a 14-year population study. J Cardiovasc Med (Hagerstown). 2016:17(1):37-43.

3. He S, Cao Y, Qin W, Chen W, Yin L, Chai H, Tao Z, Tang S, Qiu Z, Chen X. Prevalence of primary cardiac tumor malignancies in retrospective studies over six decades: a systematic review and meta-analysis. Oncotarget. 2017;8(26):43284-94.

4. Araoz PA, Eklund HE, Welch TJ, Breen JF. CT and MR imaging of primary cardiac malignancies. Radiographics. 1999;19(6):1421-34.

5. Ibrahim A, Luk A, Singhal P, Wan B, Zavodni A, Cusimano RJ, Butany J. Primary intimal (spindle cell) sarcoma of the heart: a case report and review of the literature. Case Rep Med. 2013;2013:461815.

6. Poterucha TJ, Kochav J, O'Connor DS, Rosner GF. Cardiac tumors: clinical presentation, diagnosis, and management. Curr Treat Options Oncol. 2019;20(8):66.

7. Italiano A, Bellera C, D'Angelo S. PD1/PD-L1 targeting in advanced softtissue sarcomas: a pooled analysis of phase II trials. J Hematol Oncol. 2020;13(1):55.

8. Sultan I, Bianco V, Habertheuer A, Kilic A, Gleason TG, Aranda-Michel E, Harinstein ME, Martinez-Meehan D, Arnaoutakis G, Okusanya O. Long-term outcomes of primary cardiac malignancies: multi-institutional results from the national cancer database. J Am Coll Cardiol. 2020;75(18):2338-47.

9. Moeri-Schimmel R, Pras E, Desar I, Krol S, Braam P. Primary sarcoma of the heart: case report and literature review. J Cardiothorac Surg. 2020;15(1):104.

10. Abu SW, Ramlawi B, Shapira OM, Al JO, Ravi V, Benjamin R, Durand JB, Leja MJ, Blackmon SH, Bruckner BA, Reardon MJ. Improved outcomes with the evolution of a neoadjuvant chemotherapy approach to right heart sarcoma. Ann Thorac Surg. 2017;104(1):90-6.

11. Lestuzzi C, De Paoli A, Baresic T, Miolo G, Buonadonna A. Malignant cardiac tumors: diagnosis and treatment. Future Cardiol. 2015;11(4):485-500.

12. Kassi M, Polsani V, Schutt RC, Wong S, Nabi F, Reardon MJ, Shah DJ. Differentiating benign from malignant cardiac tumors with cardiac magnetic resonance imaging. J Thorac Cardiovasc Surg. 2019;157(5):1912-22.

13. Lestuzzi C. Primary tumors of the heart. Curr Opin Cardiol. 2016;31(6):593-8

14. Lestuzzi C, Reardon MJ. Primary cardiac malignancies: the need for a multidisciplinary approach and the role of the cardio-oncologist. J Am Coll Cardiol. 2020;75(18):2348-51.

15. Blackmon SH, Patel A, Reardon MJ. Management of primary cardiac sarcomas. Expert Rev Cardiovasc Ther. 2008;6(9):1217-22.

16. Gosalakkal JA, Sugrue DD. Malignant melanoma of the right atrium: antemortem diagnosis by transvenous biopsy. Br Heart J. 1989;62(2):159-60.

17. Sze DY, Lee DP, Hofmann LV, Petersen B. Biopsy of cardiac masses using a stabilized intracardiac echocardiography-guided system. J Vasc Interv Radiol. 2008;19(11):1662-7.

18. Scott PJ, Ettles DF, Rees MR, Williams GJ. The use of combined transoesophageal echocardiography and fluoroscopy in the biopsy of a right atrial mass. Br J Radiol. 1990;63(747):222-4.

19. Schwinger ME, Katz E, Rotterdam H, Slater J, Weiss EC, Kronzon I. Right atrial papillary fibroelastoma: diagnosis by transthoracic and transesophageal echocardiography and percutaneous transvenous biopsy. Am Heart J. 1989:118(5 Pt 1):1047-50.

20. Chirillo F, Risica G, Stritoni P. Mobile right atrial mass biopsy guided by biplane transesophageal echocardiography. Int J Card Imaging. 1995;11(3):201-3. 
21. Van Camp G, Abdulsater J, Cosyns B, Liebens I, Vandenbossche JL. Transesophageal echocardiography of right atrial metastasis of a hepatocellular carcinoma. Chest. 1994;105(3):945-7.

22. Rubin DC, Ziskind AA, Hawke MW, Plotnick GD. Transesophageal echocardiographically guided percutaneous biopsy of a right atrial cardiac mass. Am Heart J. 1994;127(4 Pt 1):935-6.

23. Starr SK, Pugh DM, O'Brien-Ladner A, Stites S, Wilson DB. Right atrial mass biopsy guided by transesophageal echocardiography. Chest. 1993;104(3):969-70.

24. Salka S, Siegel R, Sagar KB. Transvenous biopsy of intracardiac tumor under transesophageal echocardiographic guidance. Am Heart J. 1993;125(6):1782-4.

25. Azuma T, Ohira A, Akagi H, Yamamoto T, Tanaka T. Transvenous biopsy of a right atrial tumor under transesophageal echocardiographic guidance. Am Heart J. 1996;131(2):402-4.

26. Segar DS, Bourdillon PD, Elsner G, Kesler K, Feigenbaum H. Intracardiac echocardiography-guided biopsy of intracardiac masses. J Am Soc Echocardiogr. 1995:8(6):927-9.

27. Malouf JF, Thompson RC, Maples WJ, Wolfe JT. Diagnosis of right atrial metastatic melanoma by transesophageal echocardiographic-guided transvenous biopsy. Mayo Clin Proc. 1996;71(12):1167-70.

28. Hammoudeh AJ, Chaaban F, Watson RM, Millman A. Transesophageal echocardiography-guided transvenous endomyocardial biopsy used to diagnose primary cardiac angiosarcoma. Cathet Cardiovasc Diagn. 1996;37(3):347-9.

29. Lynch M, Clements SD, Shanewise JS, Chen CC, Martin RP. Right-sided cardiac tumors detected by transesophageal echocardiography and its usefulness in differentiating the benign from the malignant ones. Am J Cardiol. 1997:79(6):781-4

30. Savoia MT, Liguori C, Nahar T, Marboe C, Weinberger J, Di Tullio MR, Homma S. Transesophageal echocardiography-guided transvenous biopsy of a cardiac sarcoma. J Am Soc Echocardiogr. 1997;10(7):752-5.

31. Fischer JL, Antoni D, Quadbeck J, Kramer H, Tiefenbock G, Aschenbrenner G, Strohm H, Krieg HJ, Fuchs C, Gross S, Schlemmer M, Nibler K, Garbrecht M, Henselmann L, Bultmann B. High malignant B-cell non-Hodgkin lymphoma of the heart: intravital diagnosis by transesophageal echocardiography controlled biopsy. Z Kardiol. 1997;86(10):848-56.

32. Unger $P$, Kentos $A$, Cogan $E$, Renard M, Crasset V, Stoupel E. Primary cardiac lymphoma: diagnosis by transvenous biopsy under transesophageal echocardiographic guidance. J Am Soc Echocardiogr. 1998;11(1):89-91.

33. Marti V, Aymat RM, Garcia J, Guiteras P, Carreras F, Auge JM, Cladellas M. Transthoracic echo-guided transvenous biopsy of a right atrial mass. Cathet Cardiovasc Diagn. 1998;45(3):349-50.

34. Nitta R, Sakomura Y, Tanimoto K, Hidai T, Kasanuki H, Aomi S, Nishikawa T. Primary cardiac angiosarcoma of the right atrium undiagnosed by transvenous endocardial tumor biopsy. Intern Med. 1998;37(12):1023-6.

35. Nardi P, Gaspardone A, Chiariello L, Pellegrino A, Chen LW, Orlandi A. Percutaneous transvenous biopsy for the diagnosis of a right atrial myxoma. G Ital Cardiol. 1999;29(3):308-11.

36. Galrinho A, Ferreira D, Loureiro J, Terras F, Silva S, Ferreira R. Diagnosis of a cardiac mass-a case report. Rev Port Cardiol. 2000;19(2):243-8.

37. Burling F, Devlin G, Heald S. Primary cardiac lymphoma diagnosed with transesophageal echocardiography-guided endomyocardial biopsy. Circulation. 2000;101(17):E179-81.

38. Jurkovich D, de Marchena E, Bilsker M, Fierro-Renoy C, Temple D, Garcia H. Primary cardiac lymphoma diagnosed by percutaneous intracardiac biopsy with combined fluoroscopic and transesophageal echocardiographic imaging. Catheter Cardiovasc Interv. 2000;50(2):226-33.

39. Oishi Y, Okamoto M, Sueda T, Hashimoto M, Karakawa S, Akita T, Ohkura M. Cardiac tumor biopsy under the guidance of intracardiac echocardiography. Jpn Circ J. 2000;64(8):638-40.

40. Chan KL, Veinot J, Leach A, Bedard P, Smith S, Marquis JF. Diagnosis of left atrial sarcoma by transvenous endocardial biopsy. Can J Cardiol. 2001;17(2):206-8.

41. Keller DI, Hunziker P, Buser P. Biopsy of right atrial angiosarcoma guided by transesophageal echocardiography. J Am Soc Echocardiogr. 2002;15(5):475-7.

42. Kang SM, Rim SJ, Chang HJ, Choi D, Cho SY, Cho SH, Chung N. Primary cardiac lymphoma diagnosed by transvenous biopsy under transesophageal echocardiographic guidance and treated with systemic chemotherapy. Echocardiography. 2003;20(1):101-3.
43. Flox CA, Hernandez HF, Salguero BR, Sanchez PI, Carbonell PA, Tascon PJ. Primary cardiac lymphoma: diagnosis by transjugular biopsy. Rev Esp Cardiol. 2003;56(11):1141-4

44. Scholte AJ, Frissen PH, van der Wouw PA. Transesophageal echocardiography-guided transvenous biopsy of an intracardiac tumor. Echocardiography. 2004;21(8):721-3.

45. Aqel R, Dobbs J, Lau Y, Lloyd S, Gupta H, Zoghbi GJ. Transjugular biopsy of a right atrial mass under intracardiac echocardiographic guidance. J Am Soc Echocardiogr. 2006;19(8):1072-5.

46. Mitchell AR, Timperley J, Hudsmith L, Neubauer S, Bashir Y. Intracardiac echocardiography to guide myocardial biopsy of a primary cardiac tumour. Eur J Echocardiogr. 2007;8(6):505-6.

47. Abramowitz Y, Hiller N, Perlman G, Admon D, Beeri R, Chajek-Shaul T, Leibowitz D. The diagnosis of primary cardiac lymphoma by right heart catheterization and biopsy using fluoroscopic and transthoracic echocardiographic guidance. Int J Cardiol. 2007;118(2):e39-40.

48. Kozelj M, Zorman D, Mrevlje B, Cernelc P, Zver S. Cardiac granulocytic sarcoma diagnosed by intracardiac echocardiography-guided biopsy. Int J Hematol. 2008;88(1):101-3.

49. Kutyifa V, Merkely B, Pozsonyi Z, Hosszu K, Szilagyi S, Balazs G, Toth A, Sarman P, Geller L. Intracardiac echocardiography-guided cardiac tumor mass biopsy. Orv Hetil. 2008;149(39):1857-9.

50. Higo T, Takemoto M, Ogawa K, Inoue S, Eshima K, Tada H, Sunagawa K. Intracardiac echocardiography-guided cardiac tumor biopsy. Circ J. 2009;73(2):381-3.

51. Brueck M, Bandorski D, Stahl U, Kramer W. Superior vena cava syndrome by cardiac tumor. Med Klin (Munich). 2009;104(1):52-7.

52. Kanjwal K, Colyer WJ. Transthoracic echocardiography-guided biopsy of a metastatic endometrial adenocarcinoma in the right atrium: a review of diagnosis and treatment of cardiac masses. Am J Ther. 2010;17(4):e118-25.

53. Kuppahally SS, Litwin SE, Michaels AD. Endomyocardial biopsy of right atrial angiosarcoma guided by intracardiac echocardiography. Cardiol Res Pract. 2010;2010:681726.

54. Hosokawa Y, Kodani E, Kusama Y, Kamiya M, Yoshikawa M, Hirasawa Y, Nakagomi A, Atarashi H, Maeda S, Mizuno K. Cardiac angiosarcoma diagnosed by transvenous endomyocardial biopsy with the aid of transesophageal echocardiography and intra-procedural consultation. Int Heart J. 2010;51(5):367-9.

55. Kamiya K, Sakakibara M, Yamada S, Tan M, Furihata T, Kubota K, Tsutsui H. Diffuse large B-cell lymphoma diagnosed by intracardiac echocardiography-guided cardiac tumor biopsy. Intern Med. 2012;51(9):1043-7.

56. Satya K, Kalife G, Navarijo J, Rasekh A, Wilson JM. Transseptal biopsy of a left atrial mass with 3-dimensional transesophageal echocardiographic guidance. Tex Heart Inst J. 2012;39(5):707-10.

57. Konecny T, Reeder G, Noseworthy PA, Konecny D, Carney JA, Asirvatham SJ. Percutaneous ablation and retrieval of a right atrial myxoma. Heart Lung Circ. 2014;23(11):e244-7.

58. Park KI, Kim MJ, Oh JK, Lee JH, Park JH, Choi SW, Jeong JO, Seong IW. Intracardiac echocardiography to guide biopsy for two cases of intracardiac masses. Korean Circ J. 2015;45(2):165-8.

59. Azzalini L, de Hemptinne Q, Asgar A, Ibrahim R. Right atrial mass in a patient with breast cancer: percutaneous transcatheter biopsy under intracardiac echocardiography guidance. BMJ Case Rep. 2016;2016:10-1136.

60. Schneider CM, Buiatti A, Schwamborn K, Dirschinger RJ. Diagnosis of a rare cardiac human herpesvirus-8 positive B-cell lymphoma manifestation: a case report of a transoesophageal echocardiography-guided trans-septal catheter biopsy. Eur Heart J Case Rep. 2018;2(2):y37.

61. Maeda R, Naito D, Adachi A, Shiraishi H, Sakamoto T, Matoba S. IgG4related disease involving the cardiovascular system: an intracardiac mass and a mass lesion surrounding a coronary artery. Intern Med. 2019;58(16):2363-6.

62. Naruse G, Kawasaki M, Yanase K, Tanaka T. Primary angiosarcoma in the right atrium diagnosed by a cardiac tumor biopsy using intracardiac echocardiography. J Med Ultrasound. 2020;28(2):120-2.

\section{Publisher's Note}

Springer Nature remains neutral with regard to jurisdictional claims in published maps and institutional affiliations. 